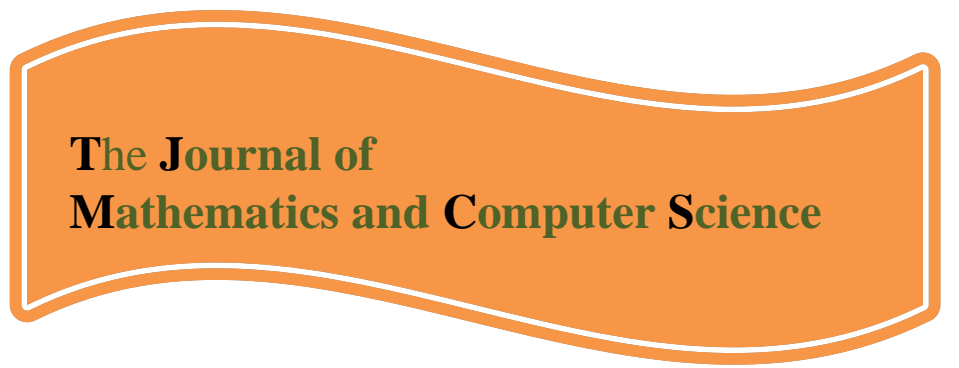

Available online at

http://www.TJMCS.com

The Journal of Mathematics and Computer Science Vol .2 No.1 (2011) 9-19

\title{
A Multi-Objective Goal Programming Approach to a Fuzzy Transportation Problem: The Case of a General Contractor Company
}

\author{
Hossein Abdollahnejad Barough1, 2* \\ hosseinab@kayson-ir.com
}

Received: October 2010, Revised: December 2010

Online Publication: January 2011

\begin{abstract}
In this paper, the author presented a transportation problem with Non-Linear constraints in which supplies and demands are trapezoidal fuzzy values and the objective function assumes multiple objectives. Then, Non-Linear constraints are linearized by defining and adding auxiliary constraints. Finally, the optimal solution of the problem is founded by solving the linear programming problem with fuzzy and crisp constraints and applying fuzzy programming technique. The method proposed to solve this problem is illustrated through numerical examples. Multi-objective goal programming methodology is used for numerical examples. The results of this research were developed and used as one of the Decision Support System models in Logistics Department of Kayson Co.
\end{abstract}

Keywords: Fuzzy Transportation Problem, Non-Linear Programming, Fuzzy Constraints, Multiobjective Goal Programming, Linear Programming.

\section{Introduction}

The classical transportation problem refers to a special class of linear programming problems. In a typical transportation problem, a product is to be transported from $\mathrm{M}$ sources to $\mathrm{N}$ destinations and their demand and supply values are $\mathrm{a}_{1}, \mathrm{a}_{2}, \ldots, \mathrm{a}_{\mathrm{M}}$ and $\mathrm{b}_{1}, \mathrm{~b}_{2}, \ldots, \mathrm{b}_{\mathrm{N}}$ respectively. In addition, there is

\footnotetext{
$1,{ }^{*}$ Corresponding author: Department of Industrial Engineering, Payam-e-Noor University..

$2,{ }^{*}$ System Analyst, Planning and System Department, Kayson Co
} 
a penalty $\mathrm{c}_{\mathrm{ij}}$ associated with transporting a unit of the product from source i to destination $\mathrm{j}$. This penalty may be cost or delivery time or safety of delivery, etc. In practice, the parameters of transportation problem i.e. demand and supply values are not always exactly known and stable. This paper deals with the case when the penalties are known exactly, but the estimate of the demand and supply values are trapezoidal fuzzy values and assuming multiple objectives. The main approaches to decision making under imprecision includes stochastic programming and fuzzy programming.

First introduced by Zadeh [19], the concept of the fuzzy set theory is used for solving different types of Linear Programming (LP) problems. Zimmermann [20] first introduced Fuzzy Linear Programming (FLP) as conventional LP. He used linear membership functions and the minimizing operator as an aggregator for these functions, and assigned an equivalent LP to fuzzy linear programming problem. Subsequently, Zimmermann's fuzzy linear programming has developed into several fuzzy optimization methods for solving the transportation problems. He presented a fuzzy approach to

multi-objective linear programming problems [22]. He also studied the duality relations in fuzzy linear programming [22]. Fuzzy linear programming problem with fuzzy coefficients was formulated by Negoita [16] and called robust programming. Dubois and Prade [13] investigated linear fuzzy constraints. Tanaka and Asai [16] also proposed a formulation of fuzzy linear programming with fuzzy constraints and gave a method for its solution which bases on inequality relations between fuzzy numbers.

Also, Chanas and Kuchta [6] proposed the concept of the optimal solution for the transportation problem with fuzzy cost coefficients expressed as L-R fuzzy numbers, and developed an algorithm for obtaining the optimal solution.Additionally, Chanas and kuchta [7] designed an algorithm for solving integer fuzzy transportation problem with fuzzy demand and supply values in the sense of maximizing the joint satisfaction of the fuzzy goal and the constraints. The term 'Goal Programming' was introduced by Charnes and Cooper [12] in 1961. Decision makers sometimes set such goals, even when they are unattainable within the available resources. Such problems are tackled with the help of the techniques of goal programming. Any constraint incorporated is called a goal. Whether the goals are attainable or not, the objective function is stated in such a way that it's optimization means as 'close as possible' to the indicated goals. Multi-objective linear programming problems exist in many managerial decision making problems. Hiller and Lieberman [14] and Ravindran et. al [17] have considered a mathematical model in which an appropriate constraint is to be chosen using binary variables. A method for modeling the multi-objective goal programming problem, using the multiplicative terms of binary variables to handle the multiple aspiration levels was presented by Chang [9]. He has also given a method where the multiplicative terms of the binary variables are replaced by a continuous variable [11].

In this paper, two goal programming models, where the multiple aspiration levels of the cost goal are handled by the use of multiplicative terms of the binary variables and by the use of a continuous variable for a transportation problem with fuzzy demand and supply values are formulated.

The organization of this paper is as follows. Section two reviews some basic definitions and assumptions of the area of fuzzy theory. Two transportation models and their respective goal programming formulations are presented in section three. Section four explains the method of solution of the linear programming problem with fuzzy and crisp constraints. Two numerical examples on the proposed method are presented in section five, these numerical examples are modified real problems in the Logistics Department of Kayson Co. and finally section six gives some concluding remarks on the proposed method.

\section{Definitions and Assumptions}


In this section, some basic notions of the area of fuzzy theory that have been defined by Kaufmaan and Gupta (1985) and Zimmermann (1996) are introduced.

Definition1: Let $\mathrm{R}$ be the space of real numbers. A Fuzzy set $\tilde{A}_{i}$ is a set of ordered pairs $\left\{\left(x, \mu_{\widetilde{A}_{i}}(x) \mid x \in R\right)\right\}$, where $\mu_{\widetilde{A}_{i}}(x): \rightarrow[0,1]$ and is upper semi continuous. Function $\mu_{\widetilde{A}_{i}}(x)$ is called membership function of the fuzzy set.

Definition 2: A convex fuzzy set, $\tilde{\mathrm{A}}_{\mathrm{i}}$, is a fuzzy set in which:

$\forall \mathrm{x}, \mathrm{y} \in \mathrm{R}, \forall \lambda \in[0,1]$,

$\mu_{\widetilde{A}_{i}}(\lambda x+(1-\lambda) y) \geq \min \left[\mu_{\widetilde{A}_{i}}(x), \mu_{\widetilde{A}_{i}}(y)\right]$

Definition3: A fuzzy set $\tilde{A}$ is called positive if its membership function is such that $\mu_{\widetilde{A}_{i}}(x)=0, \forall x \leq$ 0 .

Definition4: Trapezoidal Fuzzy Number (TFN) is a convex fuzzy set which is defined as $\tilde{A}=\left(x, \mu_{\tilde{A}_{i}}(x)\right)$ Where:

$\mu_{\widetilde{\mathrm{A}}_{\mathrm{i}}}(x)=\left\{\begin{array}{c}0, \quad x \leq a_{i}^{1} \\ \frac{\left(x-a_{i}^{1}\right)}{\left(a_{i}^{2}-a_{i}^{1}\right)}, a_{i}^{1}<x \leq a_{i}^{2} \\ 1, \quad a_{i}^{2}<x \leq a_{i}^{3} \\ \frac{\left(a_{i}^{4}-x\right)}{\left(a_{i}^{4}-a_{i}^{3}\right)}, a_{i}^{3}<x \leq a_{i}^{4} \\ 0, \quad x>a_{i}^{4}\end{array}\right.$

For convenience, TFN represented by four real parameters $a_{1}, a_{2}, a_{3}, a_{4}$ which are $\left(a_{i}^{1} \leq a_{i}^{2} \leq\right.$ $a_{i}^{3} \leq a_{i}^{4}$ ) Will be denoted by tetraploid $a_{i}^{1}, a_{i}^{2}, a_{i}^{3}, a_{i}^{4}$ (Fig.1).

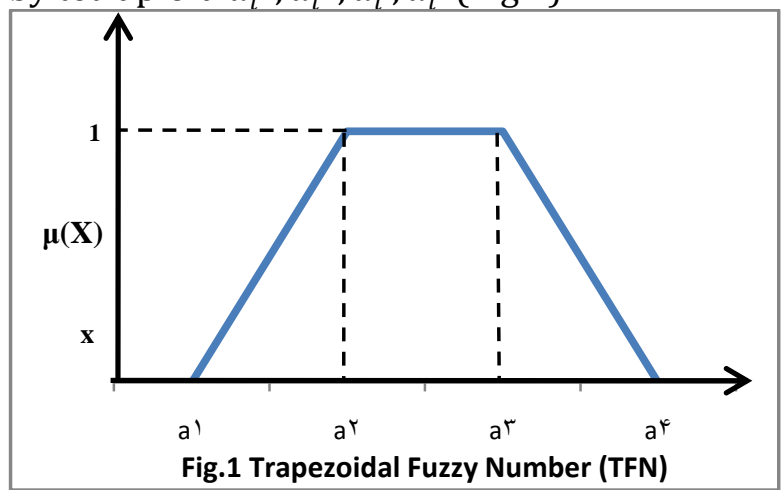

Definition5: A Trapezoidal fuzzy number $\widetilde{\mathrm{A}}=\left(a_{i}^{1}, a_{i}^{2}, a_{i}^{3}, a_{i}^{4}\right)$ is called positive TFN if: $0 \leq a_{i}^{1} \leq a_{i}^{2} \leq a_{i}^{3} \leq a_{i}^{4}$

\section{Mathematical Models}

\subsection{Notations}

The following notations are applied to describe the transportation problem:

\section{Indices and parameters:}

$\mathrm{M}$

$\mathrm{N}$

$\tilde{A}_{i}$

$\tilde{B}_{j}$
Number of sources

Number of destinations

Fuzzy quantity of $i^{\text {th }}$ source

Fuzzy quantity of $j^{\text {th }}$ destination 


$\begin{array}{ll}C_{i j} & \begin{array}{l}\text { Crisp unit of transportation cost from } \mathrm{i}^{\text {th }} \text { source to } \mathrm{j}^{\text {th }} \\ \text { destination } \\ A_{i}\end{array} \\ B_{j} & \text { Crisp quantity of } \mathrm{i}^{\text {th }} \text { source } \\ d_{i}^{+}, e^{+}, d^{+} & \text {Crisp quantity of } \mathrm{j}^{\text {th }} \text { destination } \\ d_{i}^{-}, e^{-}, d^{-} & \text {Over achievements of the } \mathrm{i}^{\text {th }} \text { goal } \\ \lambda^{*} & \text { Under achievements of the } \mathrm{i}^{\text {th }} \text { goal } \\ a, b & \text { Maximum degree of satisfaction of the fuzzy constraints } \\ \mathrm{p} & \text { Crisp numbers } \\ f^{T} & \text { Penalty of each unit of over/under achievements of } \mathrm{i}^{\text {th }} \text { goal } \\ f_{0} & \text { Objective function } \\ f_{1} & \text { Optimal value of lower bound } \lambda-c u t \\ \mu_{f} & \text { Optimal value of upper bound } \lambda-c u t\end{array}$

\section{Decision variables:}

$X_{i j}$

Crisp quantities which is transported from $\mathrm{i}^{\text {th }}$ source to $\mathrm{j}^{\text {th }}$ destination

\subsection{Transportation Linear Programming Problem}

Consider the transportation model

$$
\begin{aligned}
& \sum_{i=1}^{m} \sum_{j=1}^{n} c_{i j} x_{i j}=\left\{c_{1}, c_{2}, \ldots . . c_{k}\right\}=c\left(x_{i j}\right) \\
& \text { Subject to } \\
& \sum_{j=1}^{n} x_{i j} \cong\left(a_{i}^{1}, a_{i}^{2}, a_{i}^{3}, a_{i}^{4}\right), \quad(i=1,2, \ldots . ., m) \\
& \sum_{i=1}^{m} x_{i j} \cong\left(b_{j}^{1}, b_{j}^{2}, b_{j}^{3}, b_{j}^{4}\right), \quad(j=1,2, \ldots . ., n)
\end{aligned}
$$

$x_{i j} \geq 0$ and are integers

The objective function can assume only one of the $k$ choices (aspiration levels) $b_{1}, b_{2}, \ldots, b_{k}$. We illustrate the procedure for finding an optimal solution of the above problem for the case with four goals. Since the number of choices is $4\left(=2^{2}\right)$ two binary variables are required to model the situation. We rewrite (2) equations for four choices case as follows

$$
\begin{aligned}
& c(x)=z_{1} z_{2} c_{1}+\left(1-z_{1}\right) z_{2} c_{2}+z_{1}\left(1-z_{2}\right) c_{3}+\left(1-z_{1}\right)\left(1-z_{2}\right) c_{4}=\varphi \\
& \text { Subject to } \\
& \sum_{j=1}^{n} x_{i j} \cong\left(a_{i}^{1}, a_{i}^{2}, a_{i}^{3}, a_{i}^{4}\right), \quad(i=1,2, \ldots, m) \\
& \sum_{i=1}^{m} x_{i j} \cong\left(b_{j}^{1}, b_{j}^{2}, b_{j}^{3}, b_{j}^{4}\right), \quad(j=1,2, \ldots, n) \\
& x_{i j} \geq 0 \text { and are integers } \& z_{1}, z_{2} \text { are } 0 \text { or } 1
\end{aligned}
$$

\subsection{Multi-Objective Transportation Problem (MOTP)}

In order to minimize $\varphi$, the flexible membership function goal with the aspired level 1 (i.e. the highest possible value of membership function) is used as follows 
$\frac{\left(\text { Goal }_{\max }-\varphi\right)}{\left(\text { Goal }_{\max }-\text { Goal }_{\min }\right)}-d_{i}^{+}+d_{i}^{-}=1$

Where Goal $l_{\max }$ and Goal $_{\min }$ are respectively upper and lower bunds of the aspiration levels of the cost goal and $d_{i}^{+}$and $d_{i}^{-}$are respectively, over and under achievements of the $\mathrm{i}^{\text {th }}$ goal. Using the goal programming method presented by Chang [9] for a linear programming problem with minimization type objective function, we construct the following goal programming problem for (3) problem:

Minimize $d_{1}^{+}+d_{1}^{-}+d_{2}^{+}+d_{2}^{-}$

Subject to

$\sum_{j=1}^{n} x_{i j} \cong\left(a_{i}^{1}, a_{i}^{2}, a_{i}^{3}, a_{i}^{4}\right), \quad(i=1,2, \ldots, m)$
$\sum_{i=1}^{m} x_{i j} \cong\left(b_{j}^{1}, b_{j}^{2}, b_{j}^{3}, b_{j}^{4}\right), \quad(j=1,2, \ldots ., n)$
$\sum_{j=1}^{m} \sum_{i=1}^{n} c_{i j} x_{i j}-d_{1}^{+}+d_{1}^{-}=\varphi$
$\varphi=z_{1} z_{2} c_{1}+\left(1-z_{1}\right) z_{2} c_{2}+z_{1}\left(1-z_{2}\right) c_{3}+\left(1-z_{1}\right)\left(1-z_{2}\right) c_{4}$
$\frac{\varphi}{\left(\text { Goal }_{\text {max }}-\text { Goal }_{\text {min }}\right)}+d_{2}^{+}+d_{2}^{-}=\frac{\text { Goal }_{\text {min }}}{\left(\text { Goal }_{\text {max }}-\text { Goal }_{\text {min }}\right)}$
$x_{i j} \geq 0$ and are integers $\& z_{1}, z_{2}$ are 0 or 1

\subsection{Multi-Objective Transportation Problem (MOTP) Linearization:}

The Non-linear constraints of the above problem can be linearized by defining $\left(z_{3}=z_{1} z_{2}\right)$ and adding the linear constraint $\left(\mathrm{z}_{1}+\mathrm{z}_{2}-1 \leq 2 \mathrm{z}_{3} \leq \mathrm{z}_{1}+\mathrm{z}_{2}\right)$ in which $\mathrm{z}_{3}=0$ or 1 . Hence (5) can be written as

Minimize $d_{1}^{+}+d_{1}^{-}+d_{2}^{+}+d_{2}^{-}$

Subject to

$\sum_{j=1}^{n} x_{i j} \cong\left(a_{i}^{1}, a_{i}^{2}, a_{i}^{3}, a_{i}^{4}\right), \quad(i=1,2, \ldots . ., m)$

$\sum_{i=1}^{m} x_{i j} \cong\left(b_{j}^{1}, b_{j}^{2}, b_{j}^{3}, b_{j}^{4}\right), \quad(j=1,2, \ldots, n)$

$\sum_{j=1}^{m} \sum_{i=1}^{n} c_{i j} x_{i j}-d_{1}^{+}+d_{1}^{-}=\varphi$

$\varphi=\left(c_{2}-c_{4}\right) z_{1}+\left(c_{3}-c_{4}\right) z_{2}+\left(c_{1}-c_{2}-c_{3}-c_{4}\right) z_{3}+c_{4}$

$\frac{\varphi}{\left(\text { Goal }_{\max }-\text { Goal }_{\text {min }}\right)}+d_{2}^{+}+d_{2}^{-}=\frac{\text { Goal }_{\text {min }}}{\left(\text { Goal }_{\text {max }}-\text { Goal }_{\text {min }}\right)}$

$x_{i j} \geq 0$ and are integers $\& z_{1}, z_{2}, z_{3}$, are 0 or 1

\subsection{Strategy for Solving the Imprecise Objective Function}

Consider the following transportation model where the cost goal can assume any value in a prescribed range:

$a \leq y=c(x) \leq b$

Subject to 
$\sum_{j=1}^{n} x_{i j} \cong\left(a_{i}^{1}, a_{i}^{2}, a_{i}^{3}, a_{i}^{4}\right), \quad(i=1,2, \ldots, m)$
$\sum_{i=1}^{m} x_{i j} \cong\left(b_{j}^{1}, b_{j}^{2}, b_{j}^{3}, b_{j}^{4}\right), \quad(j=1,2, \ldots ., n)$

$x_{i j} \geq 0$ and are integers

A penalty $\mathrm{p}$ is assigned for exceeding the cost goal and there is no penalty for achieving a value lesser than the aspiration levels. Using the goal programming method given by Chang [11] for a linear programming problem with minimization type objective function, we construct the following goal programming problem.

Minimize $p\left(d^{+}\right)+e^{+}+e^{-}$

Subject to

$\sum_{j=1}^{m} \sum_{i=1}^{n} c_{i j} x_{i j}-d^{+}+d^{-}=y$

$y-e^{+}+e^{-}=a$

$a \leq y \leq b$

$d^{+}, d^{-}, e^{+}, e^{-} \geq 0$

$x_{i j} \geq 0$ and are integers

\section{Solution Representation}

(6) and (7) Problems are linear programming problems with fuzzy and crisp constraints as where $\tilde{B}_{j}=\left[b_{j}^{1}, b_{j}^{2}, b_{j}^{3}, b_{j}^{4}\right]$ is a column vector of trapezoidal fuzzy numbers. This problem can be solved by generalizing the method. The membership function of the objective function of the (8) problem can be determined by solving the following two linear programming problems.

Minimize $f^{T}\left(x_{i j}\right)$

Subject to

$\tilde{A}_{i} x_{i j} \leq a_{i}^{4}-\left(a_{i}^{4}-a_{i}^{3}\right) \lambda^{*}$

$\tilde{A}_{i} x_{i j} \geq a_{i}^{1}+\left(a_{i}^{2}-a_{i}^{1}\right) \lambda^{*}$

$A_{i} x_{i j} \leq B_{j}$

$x_{i j} \geq 0$

yielding the optimal value $f_{0}$ and

Minimize $f^{T}\left(x_{i j}\right)$

Subject to

$\tilde{A}_{i} x_{i j} \leq a_{i}^{4}$

$\tilde{A}_{i} x_{i j} \geq a_{i}^{1}$

$A_{i} x_{i j} \leq B_{j}$

$x_{i j} \geq 0$

yielding the optimal value $f_{1}$

The membership function of the objective function of problem (8) is therefore

$\mu_{\tilde{f}}\left(x_{i j}\right)=\left\{\begin{array}{cl}1 & \text {, if } f^{T}\left(x_{i j}\right) \leq f_{0} \\ \frac{\left(f^{T}\left(x_{i j}\right)-f_{1}\right)}{\left(f_{0}-f_{1}\right)} & \text {, if } f_{0} \leq f^{T}\left(x_{i j}\right) \leq f_{1} \\ 0 & \text {, if } f^{T}\left(x_{i j}\right) \geq f_{1}\end{array}\right.$

By applying fuzzy programming technique, we get 
Maximize $\lambda$

Subject to

$\left(f_{0}-f_{1}\right) \lambda+f^{T}\left(x_{i j}\right) \geq f_{1}$

$\left(a_{i}^{4}-a_{i}^{3}\right) \lambda+\tilde{A}_{i} x_{i j} \leq a_{i}^{4}$

$-\left(a_{i}^{2}-a_{i}^{1}\right) \lambda+\tilde{A}_{i} x_{i j} \geq a_{i}^{1}$

$A_{i} x_{i j} \leq B_{j}$

$x_{i j} \geq 0$

The optimal solution of the above Linear Programming gives the optimal solution to the considered fuzzy transportation problem.

\section{Numerical Examples:}

5.1. Case with Discrete Objectives

$c\left(x_{i j}\right)=2 x_{11}+3 x_{12}+4 x_{21}+2 x_{22} \cong\{9,10,11,12\}$

Subject to

$x_{11}+x_{12} \cong[1,2,3,4]$

$x_{21}+x_{22} \cong[2,3,5,6]$

$x_{11}+x_{21} \cong[1,2,3,5]$

$x_{12}+x_{22} \cong[2,4,5,6]$

$x_{i j} \geq 0$ for $i \& j=1,2$ and are integers

The goal programming formulation using (5) is

Minimize $d_{1}^{+}+d_{1}^{-}+d_{2}^{+}+d_{2}^{-}$

Subject to

$x_{11}+x_{12} \cong[1,2,3,4]$

$x_{21}+x_{22} \cong[2,3,5,6]$

$x_{11}+x_{21} \cong[1,2,3,5]$

$x_{12}+x_{22} \cong[2,4,5,6]$

$c(X)=2 x_{11}+3 x_{12}+4 x_{21}+2 x_{22}-d_{1}^{+}+d_{1}^{-}=\varphi$

$\varphi+2 z_{1}+z_{2}=12$

$\varphi+3 d_{2}^{+}-3 d_{2}^{-}=9$

We solve the following two linear programming problems from (9) and (10)

Minimize $d_{1}^{+}+d_{1}^{-}+d_{2}^{+}+d_{2}^{-}$

Subject to

$x_{11}+x_{12} \leq 3$

$x_{11}+x_{12} \geq 2$

$x_{21}+x_{22} \leq 5$

$x_{21}+x_{22} \geq 3$

$x_{11}+x_{21} \leq 3$

$x_{11}+x_{21} \geq 2$

$x_{12}+x_{22} \leq 5$

$x_{12}+x_{22} \geq 4$

$c(X)=2 x_{11}+3 x_{12}+4 x_{21}+2 x_{22}-d_{1}^{+}+d_{1}^{-}-\varphi=0$

$\varphi+2 z_{1}+z_{2}=12$

$\varphi+3 d_{2}^{+}-3 d_{2}^{-}=9$

$x_{i j} \geq 0$ for $i \& j=1,2$ and are integers

The optimal solution of this linear programming problem is $x_{11}=2, x_{22}=4, d_{2}^{-}=4, \varphi=12$ with the optimal value of the objective function $f_{1}=1$ 
Minimize $d_{1}^{-}+3 d_{2}^{-}+3 d_{2}^{-}+3 d_{2}^{-}$

Subject to

$x_{11}+x_{12} \leq 4$

$x_{11}+x_{12} \geq 1$

$x_{21}+x_{22} \leq 6$

$x_{21}+x_{22} \geq 2$

$x_{11}+x_{21} \leq 5$

$x_{11}+x_{21} \geq 1$

$x_{12}+x_{22} \leq 6$

$x_{12}+x_{22} \geq 2$

$2 x_{11}+3 x_{12}+4 x_{21}+2 x_{22}-d_{1}^{+}+d_{1}^{-}-\varphi=0$

$\varphi+2 z_{1}+z_{2}=12$

$\varphi+3 d_{2}^{+}-3 d_{2}^{-}=9$

$x_{i j} \geq 0$ for $i \& j=1,2$ and are integers

$\varphi, d_{1}^{+}, d_{1}^{-}, d_{2}^{+}, d_{2}^{-} \geq 0, z_{1} \& z_{2}=0$ or 1

The optimal solution of this linear programming problem is $x_{12}=1, x_{21}=1, x_{22}=1, d_{2}^{-}=4, \varphi=$ 12 with the optimal value of the objective function $f_{0}=0$. Hence we have from (12)

Maximize $\lambda$

Subject to

$\lambda+d_{1}^{+}+d_{1}^{-}+d_{2}^{+}+d_{2}^{-} \geq 1$

$x_{11}+x_{12}+\lambda \leq 4$

$x_{11}+x_{12}-\lambda \geq 1$

$x_{21}+x_{22}+\lambda \leq 6$

$x_{21}+x_{22}-\lambda \geq 2$

$x_{11}+x_{21}+\lambda \leq 5$

$x_{11}+x_{21}-\lambda \geq 1$

$x_{12}+x_{22}+\lambda \leq 6$

$x_{12}+x_{22}-\lambda \geq 2$

$2 x_{11}+3 x_{12}+4 x_{21}+2 x_{22}-d_{1}^{+}+d_{1}^{-}-\varphi=0$

$\varphi+2 z_{1}+z_{2}=12$

$\varphi+3 d_{2}^{+}-3 d_{2}^{-}=9$

$x_{i j} \geq 0$ for $i \& j=1,2$ and are integers

$\varphi, d_{1}^{+}, d_{1}^{-}, d_{2}^{+}, d_{2}^{-} \geq 0, z_{1} \& z_{2}=0$ or 1

Hence the optimal solution of problem (13) is $x_{11}=2, x_{22}=4, \lambda=1, \varphi=12$.

5.2. Case with Continuous Objectives

$8 \leq c(X)=2 x_{11}+3 x_{12}+4 x_{21}+2 x_{22} \leq 10$

Subject to

$x_{11}+x_{12} \cong[1,2,3,4]$

$x_{21}+x_{22} \cong[2,3,5,6]$

$x_{11}+x_{21} \cong[1,2,3,5]$

$x_{12}+x_{22} \cong[2,4,5,6]$

$x_{i j} \geq 0$ for $i \& j=1,2$ and are integers

Penalty 2 is assigned for exceeding the cost goal. The goal programming formulation is 
Minimize $2 d^{+}+e^{+}+e^{-}$

Subject to

$x_{11}+x_{12} \cong[1,2,3,4]$

$x_{21}+x_{22} \cong[2,3,5,6]$

$x_{11}+x_{21} \cong[1,2,3,5]$

$x_{12}+x_{22} \cong[2,4,5,6]$

$2 x_{11}+3 x_{12}+4 x_{21}+2 x_{22}-d^{+}+d^{-}-y=0$

$y-e^{+}+e^{-}=8$

$8 \leq y \leq 10$

$x_{i j} \geq 0$ for $i \& j=1,2$ and are integers

$d^{+}, d^{-}, e^{+}, e^{-} \geq 0$

We solve the following two linear programming problems from (9) and (10)

Minimize $2 d^{+}+e^{+}+e^{-}$

Subject to

$x_{11}+x_{12} \leq 3$

称 + 狂 $\geq 2$

$z_{1}+z_{2} \leq 5$

$z_{1}+z_{2} \geq 3$

将 $+z_{1} \leq 3$

䐂 + 硬 $\geq 2$

$x_{2}+z_{2} \leq 5$

$z_{2}+z_{2} \geq 4$

$2 x_{1}+3 x_{2}+4 z_{1}+2 z_{2}-t d+\bar{d}-\not=0$

计 $\bar{e}+\bar{e}=8$

$8 \leq y \leq 10$

$x \geq 0$ f

$\bar{e}, \bar{e}, \bar{d}, \bar{d} \geq 0$

The optimal value of the object function is $f$

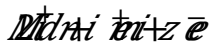

Subject to

秝 + 积 $\leq 4$

将 + 程 $\geq 1$

$z_{1}+z_{2} \leq 6$

$z_{1}+z_{2} \geq 2$

$z_{1}+z_{1} \leq 5$

$x_{1}+z_{1} \geq 1$

$\mathrm{z}_{2}+\mathrm{z}_{2} \leq 6$

$z_{2}+z_{2} \geq 2$

泝十积 $\leq 4$

$2 x_{1}+3 z_{2}+4 z_{1}+2 z_{2}-t+\bar{d}-\not=0$

$y \bar{t}+\bar{e}=8$

$8 \leq y \leq 10$

$x \geqslant 0$ 保j=1,2 aimatt e gers

$\bar{e}, \bar{e}, \bar{d}, \bar{d} \geq 0$

The optimal value of the objective function is $\varnothing=0$. Hence we have, from (12)

Mlaxi mi $z e$

Subject to

$6 \AA 2 t+t+\bar{e} \geq 6$ 


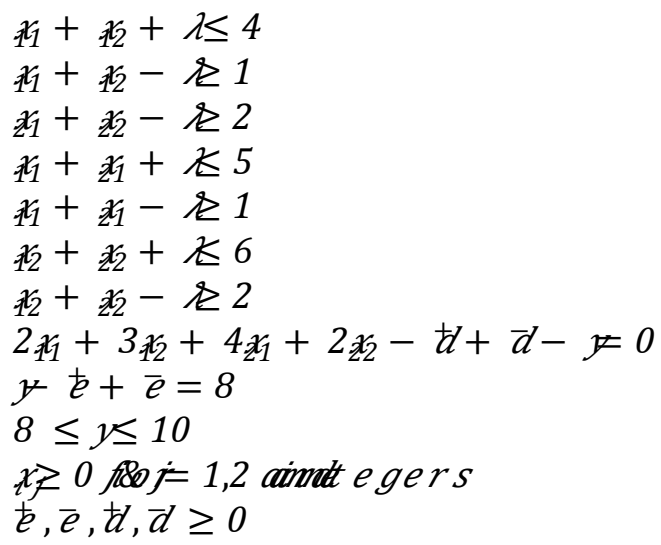

The optimal solution of problem (18) is $x_{1}=2, z_{2}=4, \not 12, \ldots 1$.

\section{Conclusion}

Transportation models have wide applications in logistics and supply chain for reducing the cost in business environments. In real world applications, the parameters in the transportation problems may not been known precisely due to uncontrollable factors. If the obtain results are crisp values it might lose some helpful information. Since the objective value is expressed by the membership function rather than by a crisp value, more information is provided for making decisions. Some previous studies have devised solution procedures for fuzzy transportation problems. The objective value derived from those studies is crisp values rather than fuzzy numbers. This study presents a MOTP with stochastic unit of transportation cost and trapezoidal fuzzy demand and supply values. In this study, the traditional transportation problem reconstructed with the multi-objective goal programming approach, then the problem linearized by defining auxiliary constraints. Finally the optimal solution of the developed model with fuzzy and crisp constraints is founded through applying fuzzy programming technique by the defined fuzzy membership function. Two numerical examples demonstrated the feasibility of applying the Multi-Objective Goal Programming approach to fuzzy transportation problems. These examples are the real modified cases in Logistics Department of Kayson Co. The results of this research were developed and used as one of the Decision Support System models in Logistics Department of Kayson Co.

\section{References}

[1] Bellman, R.R., Zadeh, L.A. "Decision making in a fuzzy environment”, Management science, Vol.17, pp.203218, 1970.

[2] Biswal, M.P., Acharya, S., "Transformation of a multi-choice linear programming problem", Applied Mathematics and Computation, 210, pp.182-188, 2009.

[3] Bit, A.K., Biswal, M.P., Alam, S.S., "Fuzzy programming approach to multi criteria decision making transportation problem", Fuzzy Sets and Systems, Vol.50, pp.135-141, 1992.

[4] Bit, A.K., Biswal, M.P., Alam, S.S., "Fuzzy programming approach to multi-objective solid transportation problem", Fuzzy Sets and Systems, Vol.57, pp.183-194, 1993.

[5] Bit, A.K., Biswal, M.P., Alam S.S., "An additive fuzzy programming model for multi-objective transportation problem", Fuzzy Sets and Systems, Vol.57, pp.313-319, 1993.

[6] Chanas, S., Kuchta, D., "A concept of the optimal solution of the transportation problem with fuzzy cost coefficients", Fuzzy Sets and Systems, Vol.82, pp.299-305, 1996.

[7] Chanas, S., Kuchta, D., "Fuzzy integer transportation problem", Fuzzy Sets and Systems, Vol.98, pp.291-298, 1998. 
[8] Chanas, S., Kolosziejczyj, W., Machaj, A., "A fuzzy approach to the transportation problem", Fuzzy Sets and Systems, Vol.13, pp.211-221, 1984.

[9] Chang, C-T., "Multi-choice goal programming", OMEGA, Vol.35, pp.389-396, 2007.

[10] Chang, C-T., "Binary fuzzy programming", European Journal of Operations Research, Vol.180, pp.29-37, 2007.

[11] Chang., C-T., "Revised Multi-choice goal programming”, Applied Mathematical Modeling, Vol.32, pp.2587$2595,2008$.

[12] Charnes, A., Cooper. W.W., "Management model and industrial application of linear programming”, Vol.1, Wiley, Newyork, 1961.

[13] Dubois, D., Prade, H., "System of linear fuzzy constraints", Fuzzy Sets and Systems, Vol.13, pp.1-10, 1982.

[14] Hiller, F., Lieberman, G., Introduction to Operations Research ,McGraw-Hill, New York, 1990.

[15] Jimenez, F., Verdegay, J.L., "Uncertain solid transportation problems", Fuzzy Sets and Systems, Vol.100, pp.45-57, 1998.

[16] Negoita, C.V., "Fuzziness in management", OPSA/TIMS, Miami, 1970.

[17] Ravindran, A., Phillips Don, T., Solberg James, J., "Operations Research Principles and Practice", second edition, John Wiles, New York, 1987.

[18] Tanaka, H., Asai, K., "Fuzzy linear programming problems with fuzzy numbers", Fuzzy Sets and Systems, Vol.13 pp.1-10, 1984.

[19] Zadeh., L.A., "Fuzzy Sets", Information and Control, Vol.8, pp.338-353, 1965.

[20] Zimmermann, H.J., "Description and optimization of fuzzy systems", International Journal of General systems , Vol.2, pp.209-215, 1976.

[21] Zimmermann, H.J., "Application of Fuzzy Set Theory to Mathematical Programming", Information Sciences, Vol.36, pp.29-58, 1985.

[22] Zimmermann, H.J., "Fuzzy mathematical programming", Computer. \& Operations Research, Vol. 10, No. 4, pp.291-298, 1983. 